\title{
Costs of gastrointestinal bleeding events in atrial fibrillation: a UK Clinical Practice Research Datalink study
}

\author{
Sreeram V Ramagopalan*,1, Mihail Samnaliev²,3, Sharada Weir² (D), Cormac J Sammon², \\ Robert Carroll ${ }^{1}$ \& Raza Alikhan ${ }^{4}$ \\ ${ }^{1}$ Centre for Observational Research \& Data Sciences, Bristol-Myers Squibb, Uxbridge, UB8 1DH, UK \\ 2PHMR Ltd, Berkeley Works, Berkley Grove, London, NW1 8XY, UK \\ ${ }^{3}$ Harvard Medical School, Boston Children's Hospital, 300 Longwood Ave, Boston, MA 02115, USA \\ ${ }^{4}$ Haemophilia \& Thrombosis Centre, University Hospital of Wales, Cardiff, CF14 4XW, UK \\ *Author for correspondence: Tel.: +44 189552 3382; Sreeram.ramagopalan@bms.com
}

Aim: To estimate the healthcare costs attributable to gastrointestinal (GI) bleeds in nonvalvular atrial fibrillation (NVAF) patients. Material \& methods: A difference-in-differences approach was used in which NVAF patients suffering a (GI) bleed were propensity score matched to those not suffering a Gl bleed, and the difference in healthcare costs in the year prior to the Gl bleed and the subsequent 3 years was compared between the two groups. Results: The mean cost attributable to Gl bleeds was $£ 3989(p<0.0001)$ in the year of the bleed and $£ 1816(p=0.001)$ in the subsequent year. Attributable costs arose primarily from inpatient visits. Conclusion: GI bleeds among NVAF patients are associated with significant healthcare costs up to 2 years following the bleed.

First draft submitted: 11 June 2019; Accepted for publication: 12 July 2019; Published online:

26 July 2019

Keywords: apixaban • Clinical Practice Research Datalink • CPRD • dabigatran • edoxaban • England • gastrointestinal bleeding $\bullet$ HES $\bullet$ Hospital Episode Statistics $\bullet$ nonvalvular atrial fibrillation $\bullet$ NVAF • oral anticoagulant • rivaroxaban • vitamin $\mathrm{K}$ antagonist

Nonvalvular atrial fibrillation (NVAF) describes an irregular heart beat in individuals who do not have moderate to severe mitral stenosis or prosthetic heart valves. The condition is associated with a five-fold increased risk of stroke [1]. Oral anticoagulant (OAC) therapy is used to reduce this risk but is associated with an increased risk of bleeding [2-4]. While the efficacy profiles of the available OACs are relatively similar, accumulating evidence suggests that the risk of bleeding may differ across them [5-9].

In order for economic assessments of OAC therapies to adequately capture the economic burden of treatment, it is important that accurate data on the costs of efficacy and safety outcomes are available, particularly if the frequency of occurrence of that outcome is expected to differ between treatments. However, limited data on the cost of GI bleeds are available, particularly in a UK setting. The data that are available relate to the initial acute costs of managing the bleed and do not capture potential longer term costs. This study therefore sought to estimate costs to the UK NHS that may be attributed to GI bleeds among patients being treated for NVAF in the 3 years following an index bleeding event.

Our aim was to provide information that will help policy makers to better understand the cost of GI bleeding and inform future economic assessments of OAC therapies in NVAF.

\section{Methods}

Study design

This incidence-based cost-of-illness study estimated the healthcare costs attributable to a GI bleed in NVAF patients by comparing total costs among individuals with NVAF who experienced GI bleeding with a matched control group of NVAF patients who did not. The primary outcome was total healthcare costs (costs associated with general practitioner [GP] consultations, inpatient stays, outpatient visits and prescriptions). Costs were estimated using a 
health system perspective and included direct medical resource use only. Approval for this study was granted by the Independent Scientific Advisory Committee for Medicines and Healthcare products Regulatory Agency on February 25, 2019 (ISAC Protocol 18-302R).

\section{Setting \& data sources}

Data were drawn from the Clinical Practice Research Datalink (CPRD)-Hospital Episode Statistics (HES) linked healthcare administrative databases. The CPRD has been previously used to describe incidence, prevalence, treatment and outcomes of NVAF in the UK [10-13]. It is comprised of primary care medical records data for about 4.4 million active (alive and registered) patients from approximately 674 general practices covering $7 \%$ of the UK population and has been shown to be representative of the demographic breakdown of the UK population. Median follow-up of 9.4 years is available for active patients [14]. As of June 2018, linkage to HES was possible for approximately $88 \%$ of the English general practices participating in CPRD Gold [15]. Hospital data on the length, type, reasons and current diagnoses for all NHS inpatient hospital admissions and outpatient clinic attendances (from 2003 onward) were captured regardless of private versus government payer or geographic residency of the patient [16].

\section{Study population}

Individuals with an atrial fibrillation (AF) diagnosis between 1 January 2012 and 1 January 2018 were identified. Those with codes indicative of valvular AF were excluded to create a cohort of NVAF patients. Individuals with a GI bleed recorded within 12 months prior to the start of follow-up were excluded to limit the study population to those at risk of suffering an incident bleed. Individuals ineligible for linkage with HES were also excluded. The study population included all remaining patients - regardless of whether or not they were treated using OAC therapy - since once a GI bleed occurs the cost is not expected to vary by therapeutic choice.

Patient follow-up began at the latest of 01/01/2012, the date of a first NVAF diagnosis, an age of 45 years, 1 year following the registration at a CPRD practice and practice up-to-standard date (point at which CPRD affirmed adequate data recording quality for the practice). Patient follow-up ended at the earliest of death, $01 / 01 / 2018$, the date a patient transferred out of the practice and the date of last data collection for the patient's practice. The study population included patients who had 12 months of data prior to their index bleed event plus data covering 1 to 3 years following the index date.

Controls were selected using propensity score matching. Logistic regression of the risk of GI bleeding among cases (NVAF patients with a bleed event) and potential control subjects (NVAF patients without a bleed event) was modeled as a function of a set of demographics, stroke risk factors, bleeding risk factors and other covariates, plus year of first eligibility in the data (to control for prior clean window for major bleeds; see Supplementary Data 1 for full list of covariates). Nearest neighbour matching on the propensity score with replacement was carried out to match each bleed case with 1 nonbleed control.

\section{Total healthcare costs}

For both cases and controls, total costs were estimated by encounter type. Standard unit cost methodology was applied to obtain costs for primary care and outpatient hospital encounters. For inpatient stays, costs were estimated based on the clinical complexity of the patient using NHS Healthcare Resource Groups (HRGs). For prescription drugs, costs were applied using the mean cost of drugs within the applicable British National Formulary (BNF) subchapter.

We took a cost-of-illness approach to estimate total healthcare costs and assigned unit costs using standard sources $[17,18]$. Primary care costs were estimated as cost per minute based on a comprehensive estimate of GP practice expenses [19] multiplied by the duration of the consultation. Outpatient attendances with and without procedures were assigned the appropriate 2017/18 unit costs from the NHS 'Reference Costs' publication [20] by treatment specialty. Inpatient care was costed using the HRG4+ 2017-18 Reference Costs Grouper software [21] before applying reference costs for each stay category from the national schedule [20]. Medications were costed based on 2017 unit costs using the first six digits of the BNF codes [22] and multiplying by the quantity prescribed.

Consistent with other studies of resource utilization [23], we estimated total annual costs per patient for the 12-month period up to the day prior to diagnosis and for each available year of follow-up. To account for inflation and variations in pricing over time, 2017 unit costs were applied to all years [19-22,24]. Total costs incorporate direct (e.g., medical staff), indirect (e.g., administration and security staff) and overhead (e.g., facilities) costs. More 
Table 1. Costing methods by encounter type.

\begin{tabular}{|c|c|}
\hline Setting & Description \\
\hline Primary care & $\begin{array}{l}\text { Unit costs per minute ( } 4 \mathrm{GBP} / \mathrm{min} \text { ) were taken from a comprehensive estimate of general practitioner practice expenses } \\
\text { (including nurses, administrative stuff, salaries, capital costs [19; Table 10.3a]. To estimate costs of primary care visits, we } \\
\text { multiplied the per minute cost by the duration (number of minutes) of each consultation recorded in CPRD GOLD. } \\
\text { Consultations with } 0 \text { duration were assigned the average for the population }\end{array}$ \\
\hline $\begin{array}{l}\text { Outpatient attendances and } \\
\text { procedures }\end{array}$ & $\begin{array}{l}\text { We distinguished between regular outpatient attendances and outpatient attendances that included an interventional } \\
\text { procedure. Unit costs for both types of attendance were taken from the NHS 'Reference Costs' publication [20] and were } \\
\text { assigned by treatment specialty and by first versus follow-up visit }\end{array}$ \\
\hline Inpatient care & $\begin{array}{l}\text { Stays in the HES inpatient dataset were classified as elective inpatient stays, long ( }>1 \text { day) nonelective inpatient stays, short } \\
\text { nonelective inpatient stays, day cases and regular day or night admissions. To allow costs to vary with clinical complexity of the } \\
\text { patient, stays were grouped by NHS HRGs, using the HRG4+2017-18 Reference Costs Grouper software [21] before applying } \\
\text { reference costs for each stay category from the national schedule [20]. This was preferred over costing by simple per diem unit } \\
\text { costs multiplied by number of inpatient days, which treats all inpatient encounters as equal in intensity of resources used. The } \\
\text { HRG grouping approach allows for the inclusion of costs associated with excess bed days. Costs for cases that were not assigned } \\
\text { to an HRG (13.4\%) were estimated using the average cost by type of service. Total inpatient costs were obtained by } \\
\text { aggregating across subcategories of inpatient care }\end{array}$ \\
\hline Prescription medications & $\begin{array}{l}\text { Each medication was costed by strength and formulation/route assigning corresponding unit costs using the first six digits of } \\
\text { the BNF codes [22] and multiplying by the quantity (e.g., number of pills) prescribed. Missing data was filled in using the mean } \\
\text { unit cost across all BNF codes }\end{array}$ \\
\hline
\end{tabular}

details on the methods used to estimate costs for each category of service (e.g., inpatient care, primary care, etc.) are found in Table 1 .

\section{Costs attributable to Gl bleeding}

In estimating the costs associated with GI bleeding in NVAF patients, we built upon the methods used in a prior study of AF bleeding in Denmark [25]. The average annual costs attributable to bleeding events were estimated in the year in which the bleeding event occurred (the incidence year) and in each of the subsequent 2 years using a matched control/regression approach [26], which identified costs attributable to the bleeding event by controlling both for typical costs of the patient themselves in a nonbleed year as well as the counterfactual of what costs would be in the analysis year for an NVAF patient in the absence of a bleeding event (represented by the control subject).

Healthcare costs were calculated for patients in the bleeding groups and control groups as the healthcare costs in year $\mathrm{t}(\mathrm{t}=1,2,3)$ after the incidence year minus the costs incurred in the year before the bleeding event (the baseline year).

Attributable healthcare costs for a year were calculated by summing up standardized costs incurred by patients in the bleeding group (individuals i) minus standardized costs incurred by patients in the control group (individuals j):

$$
C_{t}=\sum_{i j}\left[\left(C_{t i}^{d}-C_{b i}^{d}\right)-\left(C_{t j}^{c}-C_{b j}^{c}\right)\right]
$$

where $\mathrm{C}=$ costs, $\mathrm{d}=$ bleeding group, $\mathrm{c}=$ control group, $\mathrm{t}=$ post-bleed year and $\mathrm{b}=$ baseline year.

Controlling for within-person differences in the pre-post calculation eliminates between-person variations and controls for unobservable static confounders.

\section{Data analysis}

As a prelude to the cost analyses described above, simple descriptive statistics were included to illustrate characteristics of the population and trends in healthcare resource utilization for NVAF patients who experienced a clinicallyrelevant bleed versus those who did not.

All analyses were conducted using SAS software, Version 9.4 for Windows (SAS Institute, NC, USA). Differences in the matched groups were evaluated using McNemar's tests for categorical variables and sign tests for paired data for mean costs. Statistical significance of independent variables in each model were evaluated at the alpha $<0.05$ and $<0.01$ levels. All analyses were adjusted for the length of follow-up. 


\begin{tabular}{|c|c|c|c|}
\hline Variable & Cases, n (\%) & Controls, n (\%) & $p$-value \\
\hline Total & 7753 & 7753 & \\
\hline Age, years (mean) & 76.5 & 76.1 & $0.039 *$ \\
\hline Female & 3687 (47.6) & 3611 (46.6) & 0.228 \\
\hline Stroke & $1486(19.2)$ & 1509 (19.5) & 0.655 \\
\hline Thromboembolism & $113(1.5)$ & $110(1.4)$ & 0.893 \\
\hline $\mathrm{CHF}$ & 1893 (24.4) & $1888(24.4)$ & 0.940 \\
\hline Vascular & 3151 (40.6) & $3123(40.3)$ & 0.659 \\
\hline Hypertension & 6019 (77.6) & 5887 (75.9) & $0.013^{*}$ \\
\hline Diabetes Mellitus & $1713(22.1)$ & $1708(22.0)$ & 0.938 \\
\hline GI ulcer & 739 (9.5) & $778(10.0)$ & 0.304 \\
\hline Renal & 2674 (34.5) & $2623(33.8)$ & 0.397 \\
\hline Liver & $270(3.5)$ & $261(3.4)$ & 0.724 \\
\hline Heavy alcohol use & $386(5.0)$ & $386(5.0)$ & 1.000 \\
\hline NSAIDs & $682(8.8)$ & 715 (9.2) & 0.369 \\
\hline Antiplatelets & $3213(41.4)$ & 3241 (41.8) & 0.660 \\
\hline Major bleed & $2047(26.4)$ & $2043(26.4)$ & 0.956 \\
\hline Cancer & $636(8.2)$ & $658(8.5)$ & 0.542 \\
\hline Falls & $290(3.7)$ & $268(3.7)$ & 0.966 \\
\hline Paroxysmal AF & $240(3.1)$ & $257(3.3)$ & 0.466 \\
\hline OAC treatment in 90 days prior to index bleed & 2914 (37.6) & $2892(37.3)$ & 0.727 \\
\hline CHAD2DS2-VASc score (mean) & 4.0 & 4.0 & 0.103 \\
\hline Length of follow-up prior to Gl bleed in days (mean) & 468 & 459 & 0.298 \\
\hline
\end{tabular}

\section{Results}

From the CPRD Gold and linked HES databases, we identified 373,538 individuals with an AF diagnosis. A total of 241,370 were initially excluded (5748 were under 45 years of age during the follow-up period; 204,948 had no follow-up data; 23,396 had a valvular AF diagnosis; and 7278 had experienced another GI bleed event within 12 months of the start of the follow-up period). An additional 85,861 patients were excluded because either controls could not be matched to cases in the respective year or because the last date of their practice data collection was before the index date. The final study population consisted of 46,307 patients, of which 8036 were NVAF patients who had experienced a GI bleed during the study period (cases) and 38,271 were eligible to be controls because they had NVAF but no bleed event.

Cases were matched to controls using 1:1 propensity score matching. For a number of cases, it was not possible to find appropriate control subjects $(n=283)$ leaving a final sample of 7753 cases matched with 7753 controls. Table 2 shows the match results.

\section{Utilization}

We examined the percentages of individuals with any healthcare utilization by setting (primary care, inpatient, outpatient, and prescription medications) and by period (year 1, year 2, and year 3 following the index bleeding event) for cases versus controls compared with utilization in the year prior to the GI bleed (Figure 1). Percentages with utilization in all settings and all periods were higher for cases than for matched control subjects.

For both cases and controls, the percentage of individuals with utilization of primary care and drugs prescribed in a primary care or outpatient setting were somewhat lower in each year following the GI bleed compared with the year prior to the bleed.

For cases, utilization of inpatient and outpatient hospital care was higher in each year compared with the year prior to the bleed. This was particularly noticeable for inpatient care. A majority of cases had at least one inpatient stay in the year of their GI bleed (87.9\%) compared with $38.9 \%$ in the year prior to the GI bleed. In the 2 years following the GI bleed a greater proportion of patients had an inpatient stay than in the year prior to their GI bleed 

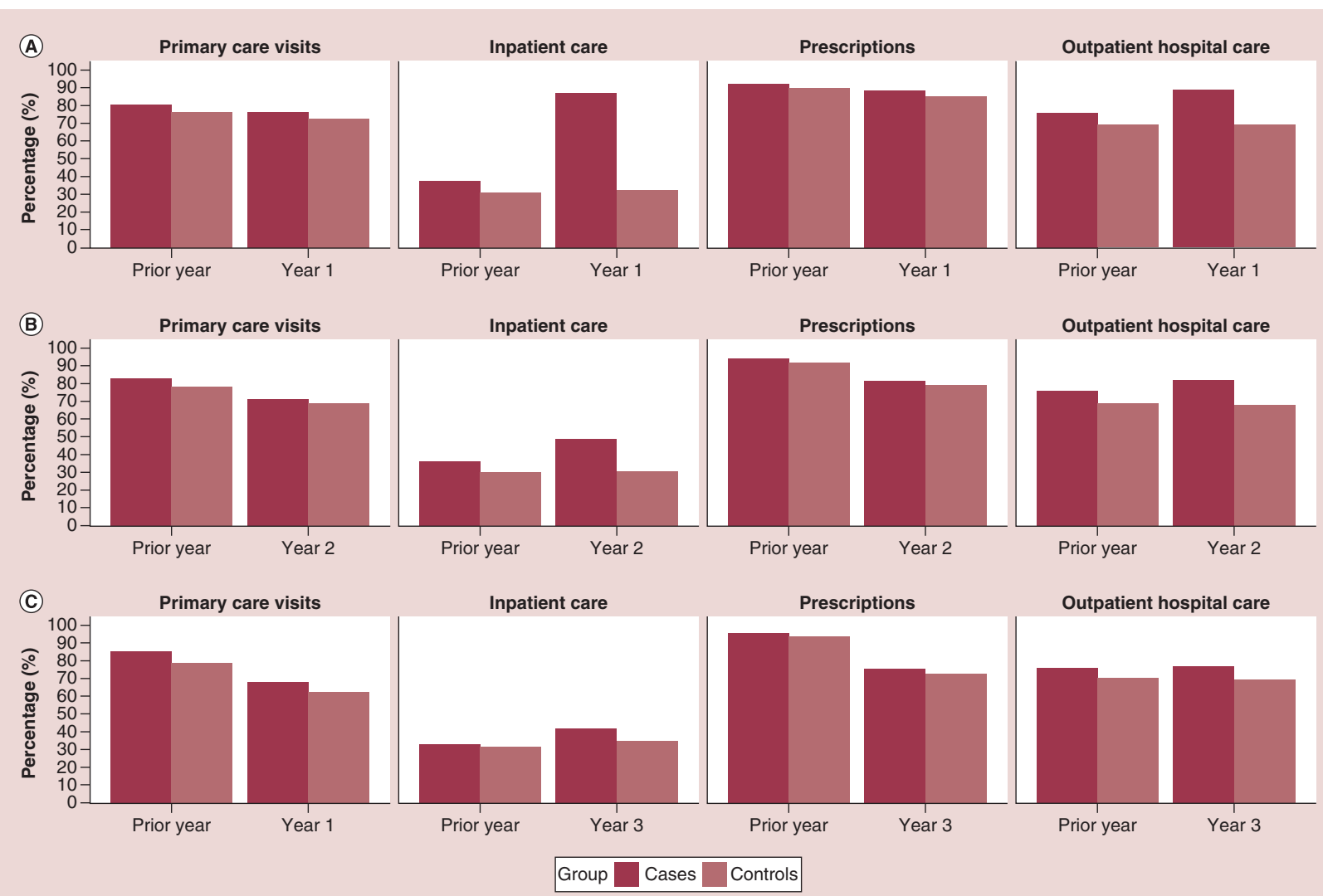

Figure 1. Healthcare utilization by setting. Percentage of cases and controls with at least one visit by setting in (A) the year of the GI bleed (year 1; $\mathrm{n}$ cases/controls = 2001), (B) the year following the Gl bleed (year 2; $\mathrm{n}$ cases/controls = 894), (C) the second year after the GI bleed (year 3; $\mathrm{n}$ cases/controls = 382). The percentages of cases and controls with at least one visit in the year prior to the Gl bleed shown for reference.

GI: Gastrointestinal.

(48.7 vs $36.4 \%$ and 43.7 vs $32.7 \%$, respectively). For controls, inpatient and outpatient utilization rate changes were much less pronounced in each of the year-over-year comparisons.

\section{Costs}

Attributable costs, overall and by healthcare setting are presented in Figure 2. Total attributable healthcare costs in the year of the GI bleed were $£ 3989$ ( $\mathrm{p}<0.0001$ ), indicating that the difference in mean costs from the year prior to the GI bleed to the year of the GI bleed in cases was significantly higher than the difference in costs observed for controls. Total attributable healthcare costs in the 2 years following the year of the GI bleed fell to $£ 1816$ ( $p=0.01)$ and $£ 1777(p=0.19)$, respectively. The largest category of attributable costs observed in the first year following the GI bleed was inpatient costs. However, outpatient prescription drug costs dominated in the second and third years (Figure 2). The absolute healthcare costs underlying the attributable costs are presented in Supplementary Data 2.

\section{Discussion}

This study examined data on NVAF patients up to 3 years from the index event of a GI bleed and represents the first attempt to examine costs of GI bleeding in NVAF patients in the UK. We are aware of only one study [25] in any jurisdiction that has considered costs beyond the index hospital admission and none that have reported on long-term follow-up data.

Using a difference-in-differences approach wherein we compared the pre/postbleed cost differential for cases who experienced a GI bleed versus controls who did not, we estimated that the mean cost attributable to the bleed in the first year alone was $£ 4075$ ( $(\mathrm{EE}=£ 361$ ), of which inpatient costs accounted for $£ 2898$ ( $S E=£ 197$ ), on average. This is in line with an estimate of $£ 2458$ ( $\mathrm{SE}=£ 216$ ) for initial inpatient costs for all patients with acute upper 


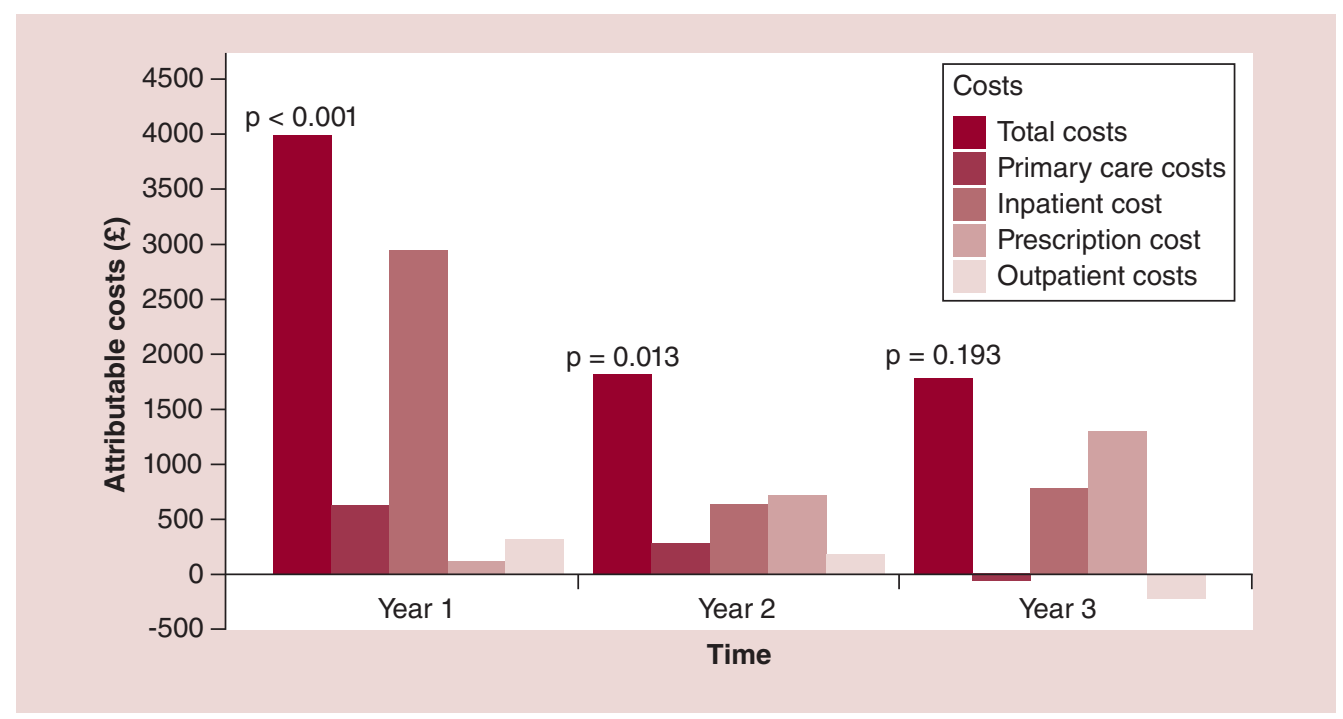

Figure 2. Attributable costs in years 1 to 3 following a gastrointestinal bleed. Attributable healthcare costs for a year were calculated as standardized costs incurred by patients in the bleeding group minus standardized costs incurred by patients in the control group; costs in each year were standardized by subtracting the costs in the year prior to the gastrointestinal bleed.

GI bleeding (not only NVAF patients) recruited from six UK hospitals in 2012/13 [27]. Our estimate is slightly higher, likely reflecting both hospital cost inflation over time and the fact that our estimate included all inpatient costs attributable to the GI bleed in the 12 months after the index event - including inpatient care necessitated by rebleed events - and not only the costs associated with the initial hospital stay for the index bleed.

Putting our results into the context of a systematic review of upper and lower GI bleeding events internationally, our inpatient estimate (approximately USD $\$ 3756$ or Can $\$ 5060$ ) is on the low end compared with a range of US studies but high compared with estimates for Canada [28]. Again, we would expect our estimate to be high owing to the use of more recent data and inclusion of a full year of follow-up data. The fact that ours is on the low end of the range of estimates published for the US (USD \$3180-8990) likely reflects much higher costs of hospital care in general in that jurisdiction than in the UK.

Attributable costs in our study in the year of the GI bleed (£4075; €4695) were lower than those observed using a similar methodology in Denmark (€8393) [25]. The difference observed may relate to the fact that the Danish study included major bleeds only whereas our study included clinically relevant nonmajor bleeds. However, relatively similar attributable costs were observed for the subsequent year in our study (£1332; €1535) and the Danish study (€1035), supporting our finding that costs attributable to GI bleeds arise beyond the year of the bleed [25].

The findings are notable from a payer perspective as a number of published cost-effectiveness models comparing OACs for stroke prophylaxis of NVAF in the UK have only considered the acute costs associated with the initial management of GI bleeds [29-32]. That is, the costs applied for a GI bleed in the base case of these models were sourced from the NHS reference costs and ranged from $£ 1494$ to $£ 1752$; these are considerably lower than the attributable costs presented in this study. As the models in question have included different rates of GI bleeds for the different OACs the relative cost-effectiveness of the treatments would differ had the costs they used been more reflective of those presented here. Specifically, a number of the studies have modelled vitamin $\mathrm{K}$ agonists (VKAs) and/or apixaban as having a considerably lower rate of GI bleeds than dabigatran, rivaroxaban and/or edoxaban [5-9], therefore VKAs and apixaban would have appeared more cost effective than currently reported in the literature were these higher costs applied to GI bleeds. The observed impact upon costs also suggests that there may be ongoing clinical implications for NVAF patients who experience a GI bleed. A history of bleeding events is a strong indicator of subsequent bleeding risk. Therefore, it is possible that some of the attributable costs observed in the 3 years following the index GI bleed arise due to the GI bleed increasing the risk of subsequent bleeds. Alternatively, occurrence of a bleed is likely to influence clinician perceptions of a patient's underlying bleeding risk leading them to alter subsequent treatment choices; this may have an impact on a patient's long-term risk of thromboembolic events. 
One potential limitation of the current research that may be possible to address in the future is that we did not have a sufficient sample to permit reliable longer run cost estimates by type of initial therapy. For a severe bleed event requiring reversal agents we might expect to find a substantive difference in cost between VKA and direct OACs. Future research may consider whether treatment with direct OACs vs VKA in the period prior to an index GI bleed is more costly in the long term.

The present study did not address the question of etiology of GI-bleeding, whether related to the choice of OAC therapy or by some underlying factor (e.g., neoplasm). Future research may also wish to consider whether OAC therapy exacerbates bleeding from causes that would otherwise have remained hidden to emerge later at a more advanced (possibly, more difficult to treat) stage.

Another limitation of the data source relates to potential confounding and missing data. We assumed that the lack of a code for an event means that an event did not occur. This may have introduced an element of misclassification as some of these individuals may have the relevant information recorded in free text or may not have had it recorded in their primary or secondary care record. For this reason, our findings refer exclusively to 'clinically relevant GI bleeds' and do not cover bleeding events that do not warrant inclusion in the diagnostic record. Notably, we believe it unlikely that significant clinical events went unrecorded, therefore any such events are likely to have been minor and associated with limited costs.

A range of potentially confounding variables were considered for inclusion in the propensity score. Additionally, the attributable cost approach used in the primary analysis accounted for changes in costs that were unlikely to be related to bleeding events. While there is potential for residual confounding due to covariate misclassification and the existence of unmeasured confounders, we believe that we have accounted for the majority of the key confounders in this way.

An additional specific limitation is that the costing approach we used for prescriptions was based on mean costs of drugs in a BNF subchapter rather than product specific costs. This was necessary as, to our knowledge, no reliable mapping of specific products in the CPRD product dictionary to costing data exists; the alternative of manually mapping each cost to a specific product was infeasible. The potential impact of applying mean costs depends on what drugs are most commonly prescribed in these individuals and how representative the mean subchapter costs are of the cost of these commonly prescribed drugs. A similar issue may have arisen for those inpatient stays that were missing an HRG code and instead were assigned a mean cost.

\section{Conclusion}

This study is the first to examine costs of GI bleeding in NVAF patients in the UK and one of the few in any jurisdiction to consider costs beyond the index hospitalization. Our findings strongly suggest that the economic implications of GI bleeds are considerable and go beyond the acute costs associated with the initial bleeding event.

\section{Future perspective}

As data on the comparative safety and effectiveness of OACs accumulate, key areas of clinical differentiation between the available treatments are emerging. It is important that the economic consequences of any such differences are accurately captured in order to allow payers to update recommendations regarding the most cost-effective treatment options. Our results suggest that when updating cost-effectiveness analyses of OAC treatment for stroke prophylaxis in NVAF the inclusion of longer term costs attributable to GI bleeds should be considered.

\section{Supplementary data}

To view the supplementary data that accompany this paper please visit the journal website at: www.futuremedicine.com/doi/sup $\mathrm{pl} / 10.2217 / \mathrm{fca}-2019-0033$

\section{Author contributions}

SV Ramagopalan, M Samnaliev, S Weir and CJ Sammon designed the study. M Samnaliev was responsible for data management and analysis. All authors were involved in the interpretation of results and the drafting of the manuscript.

Financial \& competing interests disclosure

This study was funded by Bristol-Myers Squibb (BMS) and Pfizer. SV Ramagopalan and R Carroll are employees of BMS. PHMR received financial support for the work from Bristol-Myers Squibb and Pfizer. The authors have no other relevant affiliations or financial involvement with any organization or entity with a financial interest in or financial conflict with the subject matter or materials discussed in the manuscript apart from those disclosed.

No writing assistance was utilized in the production of this manuscript. 
Ethical conduct of research

This study was approved by the Independent Scientific Advisory Committee (ISAC) with CPRD number 18-302R.

Data sharing

CPRD data were provided under a license that does not permit sharing. Data are, however, obtainable directly from CPRD under their standard conditions

\section{Open Access}

This work is licensed under the Attribution-NonCommercial-NoDerivatives 4.0 Unported License. To view a copy of this license, visit http://creativecommons.org/licenses/by-nc-nd/4.0/

\section{Summary points}

- Existing evidence suggests that the risk of gastrointestinal (GI) bleeds differs across the available oral anticoagulant treatment options.

- There are limited data on the longer term economic implications of GI bleeds.

- We sought to estimate the healthcare costs attributable to GI bleeds in nonvalvular atrial fibrillation (NVAF) patients in England in the 3 years following a Gl bleed.

- A difference-in-differences approach was used in which NVAF patients suffering a GI bleed were propensity score matched to those not suffering a GI bleed.

- The difference in healthcare costs in the year prior to the Gl bleed and the subsequent three years was then compared between the two groups to calculate the cost attributable to a GI bleed.

- The mean cost attributable to GI bleeds was $£ 3989$ ( $p<0.0001)$ in the year of the GI bleed and $£ 1816(p=0.01)$ in the subsequent year.

- These costs are higher than those used in previous cost-effectiveness analyses which have primary focused on acute inpatient costs.

- The inclusion of longer term costs attributable to GI bleeds in cost-effectiveness analyses of oral anticoagulants in NVAF should be considered.

\section{References}

1. Wolf PA, Abbott RD, Kannel WB. Atrial fibrillation as an independent risk factor for stroke: the Framingham Study. Stroke 22(8), 983-988 (1991).

2. Cope S, Clemens A, Hammès F, Noack H, Jansen JP. Critical appraisal of network meta-analyses evaluating the efficacy and safety of new oral anticoagulants in atrial fibrillation stroke prevention trials. Value Health 18(2), 234-249 (2015).

3. Assiri A, Al-Majzoub O, Kanaan AO, Donovan JL, Silva M. Mixed treatment comparison meta-analysis of aspirin, warfarin, and new anticoagulants for stroke prevention in patients with nonvalvular atrial fibrillation. Clin. Ther. 35(7), 967-984.e2 (2013).

4. Dogliotti A, Paolasso E, Giugliano RP. Current and new oral antithrombotics in non-valvular atrial fibrillation: a network meta-analysis of 79,808 patients. Heart 100(5), 396-405 (2014).

5. Abraham NS, Noseworthy PA, Yao X et al. Gastrointestinal safety of direct oral anticoagulants: a large population-based study. Gastroenterology 152(5), 1014-1022.e1 (2017).

6. Rivera-Caravaca JM, Lip GYH. Apixaban may have lower risk of GI bleeding compared with dabigatran and rivaroxaban in patients with atrial fibrillation. Evid. Based. Med. 22(4), 154-155 (2017).

7. Cheung KS, Leung WK. Gastrointestinal bleeding in patients on novel oral anticoagulants: risk, prevention and management. World J. Gastroenterol. 23(11), 1954-1963 (2017).

8. Cohen AT, Hill NR, Luo X, Masseria C, Abariga SA, Ashaye AO. A systematic review of network meta-analyses among patients with nonvalvular atrial fibrillation: A comparison of efficacy and safety following treatment with direct oral anticoagulants. Int. J. Cardiol. 269, 174-181 (2018)

9. Coleman CI, Briere JB, Fauchier L et al. Meta-analysis of real-world evidence comparing non-vitamin K antagonist oral anticoagulants with vitamin $\mathrm{K}$ antagonists for the treatment of patients with non-valvular atrial fibrillation. J. Mark. Access Heal. Policy 7(1), 1574541 (2019)

10. Adderley NJ, Ryan R, Nirantharakumar K et al. Prevalence and treatment of atrial fibrillation in UK general practice from 2000 to 2016 . Heart 105(1), 27-33 (2019).

11. Ramagopalan SV, Graham S, Carroll $\mathrm{R}$ et al. Discontinuation and primary care visits in nonvalvular atrial fibrillation patients treated with apixaban or warfarin. J. Comp. Eff. Res. 8(6), 371-379 (2019). 
12. Martinez C, Katholing A, Wallenhorst C, Granziera S, Cohen AT, Freedman SB. Increasing incidence of non-valvular atrial fibrillation in the UK from 2001 to 2013. Heart 101(21), 1748-1754 (2015).

13. Gallagher AM, van Staa TP, Murray-Thomas T et al. Population-based cohort study of warfarin-treated patients with atrial fibrillation: incidence of cardiovascular and bleeding outcomes. BMJ Open. 4(1), e003839 (2014).

14. Herrett E, Gallagher AM, Bhaskaran K et al. Data resource profile: Clinical Practice Research Datalink (CPRD). Int. J. Epidemiol. 44(3), $827-836$ (2015).

15. Padmanabhan S, Carty L, Cameron E, Ghosh RE, Williams R, Strongman H. Approach to record linkage of primary care data from Clinical Practice Research Datalink to other health-related patient data: overview and implications. Eur. J. Epidemiol. 34(1), 91-99 (2019).

16. NHS Digital, The Health and Social Care Information Centre. Hospital Episode Statistics: hospital outpatient activity 2011-12 summary report (2012). http://content.digital.nhs.uk/catalogue/PUB09379/hosp-outp-acti-11-12-summ-repo-rep.pdf

17. Onukwugha E, McRae J, Kravetz A et al. Cost-of-illness studies: an updated review of current methods. Pharmacoeconomics 34(1), 43-58 (2016).

18. Drummond MF, Sculpher MJ, Torrance G, O'Brien J, Stoddart GL. Methods for the Economic Evaluation of Health Care Programme. $3^{\text {rd }}$ edition. Drummond MF (Ed). Oxford, University Press, Oxford, UK, (2005).

19. Curtis L, Burns A. Unit costs of health and social care 2017, Personal social services research unit, University of Kent, Canterbury https://doi.org/10.22024/UniKent/01.02/65559 (2019).

20. Department of Health. NHS reference costs 2017/18 (2018). https://improvement.nhs.uk/resources/reference-costs/\#rc1718

21. NHS Digital. HRG4+ 2017/18 reference costs grouper and documentation (2019). https://digital.nhs.uk/services/national-casemix-office/downloads-groupers-and-tools/costing-hrg4-2017-18-reference-costs-grouper

22. British National Formulary (BNF). BNF publications homepage (2018). www.bnf.org/bnf/org_450080.htm

23. Adogwa O, Owens R, Karikari I et al. Revision lumbar surgery in elderly patients with symptomatic pseudarthrosis, adjacent-segment disease, or same-level recurrent stenosis. Part 2. A cost-effectiveness analysis: clinical article. J. Neurosurg. Spine 18(2), 147-53 (2013).

24. Glick HA, Doshi JA, Sonnad SS, Polsky D. Economic Evaluation in Clinical Trials. Polsky D (Ed.). Oxford University Press, Oxford, UK (2007).

25. Jakobsen M, Kolodziejczyk C, Klausen Fredslund E, Poulsen PB, Dybro L, Paaske Johnsen S. Costs of major intracranial, gastrointestinal and other bleeding events in patients with atrial fibrillation - a nationwide cohort study. BMC Health Serv. Res. 17(1), 398 (2017).

26. Akobundu E, Ju J, Blatt L et al. Cost-of-illness studies: a review of current methods. Pharmacoeconomics 24(9), 869-890 (2006).

27. Campbell HE, Stokes EA, Bargo D et al. Costs and quality of life associated with acute upper gastrointestinal bleeding in the UK: cohort analysis of patients in a cluster randomised trial. BMJ Open 5(4), e007230 (2015).

28. Parker DR, Luo X, Jalbert JJ, Assaf AR. Impact of upper and lower gastrointestinal blood loss on healthcare utilization and costs: a systematic review. J. Med. Econ. 14(3), 279-287 (2011).

29. Dorian P, Kongnakorn T, Phatak H et al. Cost-effectiveness of apixaban vs. current standard of care for stroke prevention in patients with atrial fibrillation. Eur. Heart J. 35(28), 1897-1906 (2014).

30. Lip GYH, Lanitis T, Kongnakorn T et al. Cost-effectiveness of apixaban compared with edoxaban for stroke prevention in nonvalvular atrial fibrillation. Clin. Ther. 37(11), 2476-2488.e27 (2015).

31. López-López JA, Sterne JAC, Thom HHZ et al. Oral anticoagulants for prevention of stroke in atrial fibrillation: systematic review, network meta-analysis, and cost-effectiveness analysis. BMJ 359, j5058 (2017).

32. Zheng Y, Sorensen SV, Gonschior A-K et al. Comparison of the cost-effectiveness of new oral anticoagulants for the prevention of stroke and systemic embolism in atrial fibrillation in a UK setting. Clin. Ther. 36(12), 2015-2028.e2 (2014). 
